ARTICLE

DOI: $10.1038 / \mathrm{s} 41467-018-02978-7$

OPEN

\title{
Efficient green light-emitting diodes based on quasi-two-dimensional composition and phase engineered perovskite with surface passivation
}

Xiaolei Yang 1,2, Xingwang Zhang 1,3, Jinxiang Deng2, Zema Chu1, Qi Jiang1,3, Junhua Meng1,3, Pengyang Wang1,3, Liuqi Zhang ${ }^{1,3}$, Zhigang Yin ${ }^{1,3} \&$ Jingbi You (1) ${ }^{1,3}$

Perovskite light-emitting diodes (LEDs) are attracting great attention due to their efficient and narrow emission. Quasi-two-dimensional perovskites with Ruddlesden-Popper-type layered structures can enlarge exciton binding energy and confine charge carriers and are considered good candidate materials for efficient LEDs. However, these materials usually contain a mixture of phases and the phase impurity could cause low emission efficiency. In addition, converting three-dimensional into quasi-two-dimensional perovskite introduces more defects on the surface or at the grain boundaries due to the reduction of crystal sizes. Both factors limit the emission efficiency of LEDs. Here, firstly, through composition and phase engineering, optimal quasi-two-dimensional perovskites are selected. Secondly, surface passivation is carried out by coating organic small molecule trioctylphosphine oxide on the perovskite thin film surface. Accordingly, green LEDs based on quasi-two-dimensional perovskite reach a current efficiency of $62.4 \mathrm{~cd} \mathrm{~A}^{-1}$ and external quantum efficiency of $14.36 \%$.

\footnotetext{
${ }^{1}$ Key Laboratory of Semiconductor Materials Science, Institute of Semiconductors, Chinese Academy of Sciences, Beijing, 100083, China. ${ }^{2}$ College of Applied Sciences, Beijing University of Technology, Beijing, 100124, China. ${ }^{3}$ College of Materials Science and Opto-electronic Technology, University of Chinese Academy of Sciences, Beijing, 100049, China. Correspondence and requests for materials should be addressed to J.Y. (email: jyou@semi.ac.cn)
} 
S olution-processed halide perovskite semiconductor materials show good photoluminescence quantum yields (PLQYs) $1-7$, which motivates applications in displays and lighting 8 . The perovskite light-emitting diodes (PeLEDs) have been reported with moderate electroluminescence (EL) efficiencies as well $^{9-15}$. Their external quantum efficiencies (EQEs) have reached about $10 \%$ for near-infrared ${ }^{13-15}$ and also in green emission $^{11,12,15}$. Hybrid organic-inorganic perovskites are better known for their free charge carriers with long diffusion length, which is good for solar cells. However, opposite properties are desired when they are used as emitting materials in LEDs ${ }^{11,16-18}$. The idea is to spatially confine charges with the insulating layers, and thus the exciton dissociation is suppressed and the radiative recombination can be enhanced in the perovskite films. Several efficient methods have been adopted, including polymer composite $^{19}$, nanocrystal pinning ${ }^{11}$ and quantum dots ${ }^{3-7}$.

The exciton binding energy is enlarged in the two-dimensional (2D) perovskite and thus the emission efficiency can be enhanced $^{20,21}$. In the early reports, $2 \mathrm{D}$ perovskite materials were used as emitting layers, while electroluminescence can only be observed at liquid nitrogen temperature ${ }^{20}$. Recently, quasi-twodimensional (quasi-2D) perovskites have been proposed by doping 2D perovskites into three-dimensional (3D) perovskite structures. The initial idea was to enhance the humidity stability of perovskite solar cells ${ }^{22-24}$. More recently, it was found that multiple quantum wells could be assembled in these quasi-2D perovskites $^{13,14}$, which can facilitate the formation of exciton and reduce the possibility of exciton dissociation. This makes the
quasi-2D perovskite an efficient luminescent material ${ }^{13-15,25}$. The working mechanism of quasi-2D PeLEDs has been fully discussed elsewhere ${ }^{13,14}$. Electrons and holes injected from large bandgap phases (small $n$-phase) can rapidly transfer into small bandgap phases (large $n$-phase), then electrons and holes recombine in large $n$-phase and emit as photons. The EQEs of quasi-2D perovskite LEDs have achieved around $10 \%{ }^{13,14}$ and highest EQE of $11.7 \%$ in near-infrared region was obtained ${ }^{14}$. Comparing to organic or inorganic LED counterparts ${ }^{26,27}$, there is still room for improvement in the EQE.

It is known that quasi-2D perovskite materials usually contain a mixture of phases and the phase impurity could lead to low emission efficiency, and thus fine control of the composition/ phases is critical for efficient emission. In addition, solution processed thin films of quasi-2D perovskite show reduced crystal size compared to those of $3 \mathrm{D}$ perovskite ${ }^{13,15}$. This could increase the concentration of the defects and traps on the film surface and grain boundaries, which will act as non-radiative recombination centers and thus decrease the emission efficiency. Surface passivation has been proved an efficient way for reducing the defects in perovskite solar cells ${ }^{28-30}$.

In this article, $\mathrm{PEA}_{2}\left(\mathrm{FAPbBr}_{3}\right)_{n-1} \mathrm{PbBr}_{4}(n=1,2, \ldots, \infty)$ is investigated as the emitting layer for quasi-2D perovskite LEDs, where PEA and FA are phenylethylammonium $\left(\mathrm{C}_{6} \mathrm{H}_{5} \mathrm{C}_{2} \mathrm{H}_{4} \mathrm{NH}_{3}{ }^{+}\right)$ and formamidinium $\left(\mathrm{HC}\left(\mathrm{NH}_{2}\right)_{2}{ }^{+}\right)$, respectively. Firstly, we optimize the films phase engineering/phase engineering. We find that the perovskite with $n=3$ composition shows decent phases and the obtained films show the highest photoluminescence (PL)

a

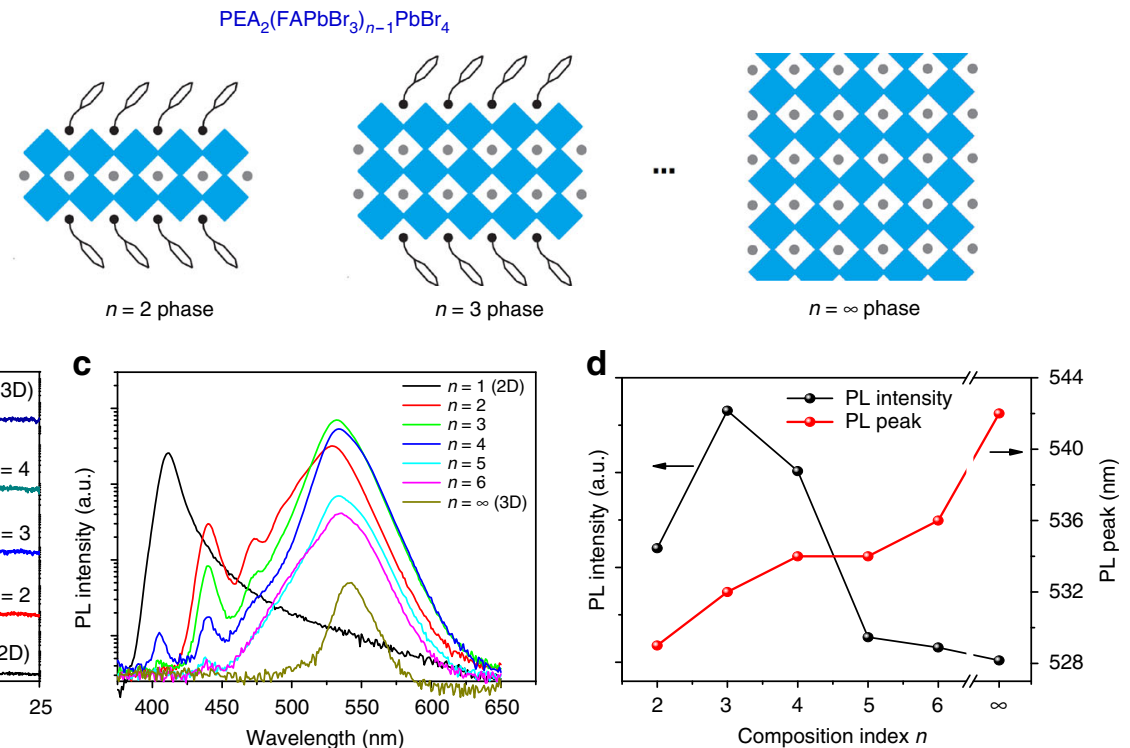

e

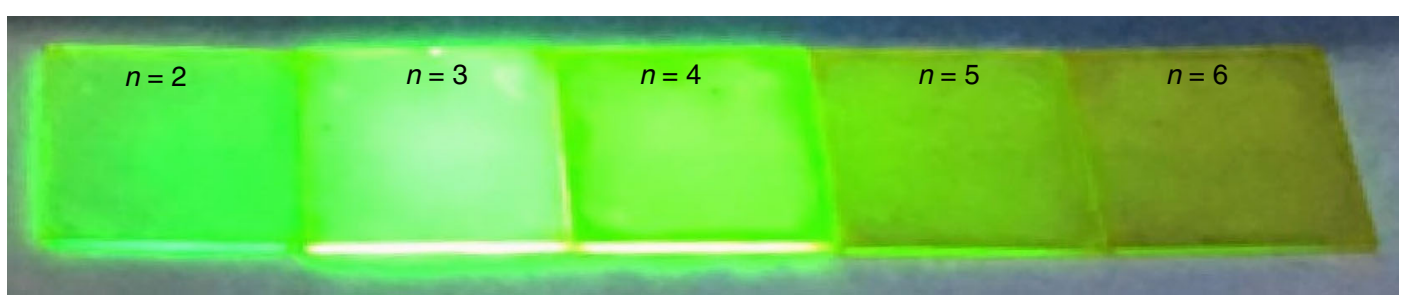

Fig. 1 Properties of perovskite films with different compositions. a Scheme of $P E A_{2}\left(F A P b B r_{3}\right)_{n-1} P\left(B r_{4}\right.$ phases. The black part is $P E A$, the blue square is $\mathrm{PbBr}_{6}$-octahedron and the gray dot is $F A$. $\mathbf{b}$ X-ray diffraction patterns of $\mathrm{PEA}_{2}(\mathrm{FAPbBr})_{n-1} \mathrm{PbBr}_{4}$ films with different $n$-compositions. Diffraction patterns of $n=1$ phase (black vertical lines), $n=2$ phase (pink vertical lines) and $n=\infty$ phase (marked with (100)) were labeled. The analysis details can be found in the Supplementary Table 2. c Photoluminescence of $\mathrm{PEA}_{2}\left(\mathrm{FAPbBr}_{3}\right)_{n-1} \mathrm{PbBr}_{4}$ with different $n$-compositions. d Photoluminescence intensity and peak of $\mathrm{PEA}_{2}\left(\mathrm{FAPbBr}_{3}\right)_{n-1} \mathrm{PbBr}_{4}$ films with different compositions with the data collected from c. e Photoluminescence image of $\mathrm{PEA}_{2}\left(\mathrm{FAPbBr}_{3}\right)_{n-1} \mathrm{PbBr}_{4}$ films with different compositions under ultraviolet lamp excitation. Figure 1a adapted from ref. 13 (copyright 2016 Macmillan Publishers) 
yield. Secondly, we present surface passivation of quasi-2D perovskite films by trioctylphosphine oxide (TOPO) treatment ${ }^{31}$. Accordingly, the PeLEDs based on $\mathrm{PEA}_{2}\left(\mathrm{FAPbBr}_{3}\right)_{n-1} \mathrm{PbBr}_{4}$ with $n=3$ composition show green emission with current efficiency (CE) of $62.4 \mathrm{~cd} \mathrm{~A}^{-1}$ and EQE of $14.36 \%$, which is the highest efficiency for PeLEDs reported so far.

\section{Results}

Properties of quasi-2D perovskite with different compositions. The schematics of $n$-phase $\mathrm{PEA}_{2}\left(\mathrm{FAPbBr}_{3}\right)_{n-1} \mathrm{PbBr}_{4}$ are drawn in Fig.1a, illustrating that the $n$-phase unit cell contains $n$ - 1 sheets of $\mathrm{PbBr}_{6}$-octahedra and two PEA tiers. The perovskite films are prepared by spin-coating a precursor solution using a crystalpinning process ${ }^{11,32}$. Their compositions are controlled by the ratio PEABr:FABr:PbBr ${ }_{2}$ (Supplementary Table 1), and compositions $n$ ranging from 2 to 6 are discussed. Previous results suggested that the quasi-2D perovskite films are not a single phase but contain a variety of $n$-phases ${ }^{13,14}$. For example, if the precursor solution with $n=2$ composition is spin-coated, the generated films might include $n=2$ phase but could also contain other phases such as $n=1$ or $n \geq 3$. To avoid misunderstanding, we distinguish the composition and phase by $n$-composition and $n$-phase, respectively. Methylammonium chloride (MACl) is also added into the precursor to improve the morphology and enhance the emission efficiency of the formed films (Supplementary Figs. 1, 2 and 12), but most MACl escape from the film during annealing ${ }^{33}$. The role of $\mathrm{MACl}$ has been well investigated in solar cells ${ }^{33,34}$. First, the $\mathrm{MACl}$ can slow down perovskite crystallization and improve film morphology. Second, the incorporation of a very small amount of elemental $\mathrm{Cl}$ could passivate the grain boundary, and then enhance the emission efficiency (Supplementary Fig. 2).

X-ray diffraction (XRD) measurements are carried out to determine the accurate phases in our obtained quasi-2D perovskite films. As shown in Fig. 1b, all the perovskite films with $n \geq 2$ compositions show diffraction peaks at $14.8^{\circ}$, which is the same as the (100) diffraction patterns of the $3 \mathrm{D}$ perovskite $\mathrm{FAPbBr}_{3}$ (refs. ${ }^{7,35}$ ). However, the peaks become much more broadened, indicating that the diffraction peak could be from a series of $\mathrm{PEA}_{2}\left(\mathrm{FAPbBr}_{3}\right)_{n-1} \mathrm{PbBr}_{4}$ phases with large $n(n \rightarrow \infty)$ or $3 \mathrm{D} \mathrm{FAPbBr} 3$ with nano-size grains (Supplementary Fig. 3$)^{15}$. This result shows that all the quasi-2D perovskite films always contain large $n$-phases $(n \rightarrow \infty)$ or $3 \mathrm{D}$ perovskite regardless of the composition of the precursor. Atomic force microscopy (AFM) images confirm the reduction of the crystal size during the transformation of 3D perovskite into quasi-2D perovskites (Supplementary Fig.3).

In addition to the diffraction peak at $14.8^{\circ}$, a series of Bragg reflections at low angles $\left(2 \theta<14.8^{\circ}\right)$ are observed for these quasi$2 \mathrm{D}$ perovskites (Fig. $1 \mathrm{~b}$ ). This indicates that the PEA group with large size is incorporated, and the size of unit cells is enlarged compared with $3 \mathrm{D}$ perovskite ${ }^{14,25,36}$. According to the analysis shown in Supplementary Table 2 and the unit cells of $n$-phases shown in Fig.la, it is found that the diffraction peaks $\left(2 \theta<14.8^{\circ}\right)$ are attributed to the $n=1$ and $n=2$ phases (diffraction patterns from $n \geq 3$ phases are not observed here). Specifically, for $n=1$ composition, only the $n=1$ phase exists; for $n=2$ composition, the $n=1$ and $n=2$ phases are both presented in the films. It is unexpected that diffraction peaks of the $n=1$ phase is absent in the $n=3$ composition, while obvious diffraction peaks from the $n=2$ phase are observed. For $n \geq 4$ compositions $(n>4$ compositions are not shown here due to very weak small $n$-phases diffractions), the diffraction peaks from small $n$-phases become weak or absent.

The photoluminescence (PL) results of the quasi-2D perovskite films with different compositions are shown in Fig. 1c, and the 2D $(n=1)$ and $3 \mathrm{D}(n=\infty)$ perovskite films are also included for comparison. The 2D perovskite shows an emission at $412 \mathrm{~nm}$ $(3.01 \mathrm{eV})$ and the long tail ${ }^{21,37}$ at a longer wavelength, which could be due to the self-trapped exciton emission ${ }^{21}$ or the disordered structure. The $3 \mathrm{D}$ perovskite showed a band edge emission peak at $542 \mathrm{~nm}(2.29 \mathrm{eV})^{38}$. All quasi-2D perovskite films have two main emission peaks: one in the green region (approximately $532 \mathrm{~nm}$, approximately $2.33 \mathrm{eV}$ ) and another in the blue region (at $440 \mathrm{~nm}, 2.82 \mathrm{eV}$ ). Comparing the $\mathrm{PL}$ with the absorption spectra (Fig.1c and Supplementary Fig. 4), it can be found that the stronger green emission at approximately $532 \mathrm{~nm}$ comes from large $n$-phases $(n \rightarrow \infty)$ or $3 \mathrm{D}$ perovskite, and the weaker blue emission at $440 \mathrm{~nm}$ comes from the $n=2$ phase. The emission phases we find are nearly almost consistent with the XRD results. There are some exceptions: for the $n=2$ composition, we have not observed the $n=1$ phase emission, while the $n$ $=4$ composition shows an emission at $405 \mathrm{~nm}$ from the $2 \mathrm{D}$ perovskite phase $(n=1)$, and the reason for this is not clear at present.

The relative PL intensity and emission peak wavelength in the green emission from the quasi-2D perovskites and 3D perovskite are plotted in Fig. 1d. It is found that the $n=3$ composition film shows the brightest green emission. The significant blue-shift of the green emission from quasi-2D perovskites is observed compared with the $3 \mathrm{D}$ perovskite. In addition, a smaller $n$ composition showed a larger blue-shift, indicating that the blueshift is due to the quantum confinement effect. The emission images of these quasi-2D perovskite films under ultraviolet excitation (Fig. 1e) also confirms that the $n=3$ composition shows the best PL.

Compared with 3D perovskites, significant enhancement of the $\mathrm{PL}$ is observed for quasi-2D perovskites (Fig.1c). This is due to the quantum confinement effect via the formation of quantum well structures between large bandgap phases (smaller $n$-phases) and small bandgap phases $(n \rightarrow \infty \text { phase or } 3 \mathrm{D} \text { perovskite })^{13,14}$. The different emissions from various compositions observed in Fig.1c could be explained by the competition between confinement phase (small $n$-phase) and emission phase (large $n$-phase, $n \rightarrow \infty$ phase). Here, we use $n=2,3,4$ compositions as examples. According to XRD results shown in Fig.1b, the $n=2$ confinement phase and $3 \mathrm{D}$ emission phase dominate in $n=2$ and $n=4$ composition, respectively, and both of these will cause weak green emission. While the amount of $n=2$ phase and $3 \mathrm{D}$ phase are adequate in the $n=3$ composition, this could be the reason of the highest PL of $n=3$ composition. The PLQY of the $n=3$ shows a value as high as $57.3 \%$ under low density excitation (excitation wavelength of $400 \mathrm{~nm}$, power density of $3.5 \mathrm{~mW} \mathrm{~cm}^{-2}$ ). The quantum well of $n=3$ composition is mainly formed by the $n=2$ and $n \rightarrow \infty$ phases (Supplementary Fig. 6).

Passivation of quasi-2D perovskite. Keeping in mind that the $n$ $=3$ composition of $\mathrm{PEA}_{2}\left(\mathrm{FAPbBr}_{3}\right)_{n-1} \mathrm{PbBr}_{4}$ perovskite could be a good candidate for an emitting material, we further improve its emission by surface passivation. An organic small molecule TOPO is spin-coated onto the perovskite surface to form a thin passivation layer ${ }^{31}$. It is found that the PLQY of the $n=3 \mathrm{com}$ position film was significantly increased from $57.3 \%$ to $73.8 \%$ after coating with the TOPO layer (Fig. 2a), confirming the passivation effect of the TOPO layer. The average fluorescence lifetime of the $n=3$ composition film is also increased from 0.17 $\mu$ s to $0.36 \mu$ s after TOPO treatment (Fig. 2b). Although a small red-shift in PL spectra has been observed for the TOPO-treated $3 \mathrm{D}$ perovskite film previously ${ }^{31}$, no obvious modulation of $\mathrm{PL}$ due to TOPO treatment is observed in this study (Supplementary Fig. 7). 

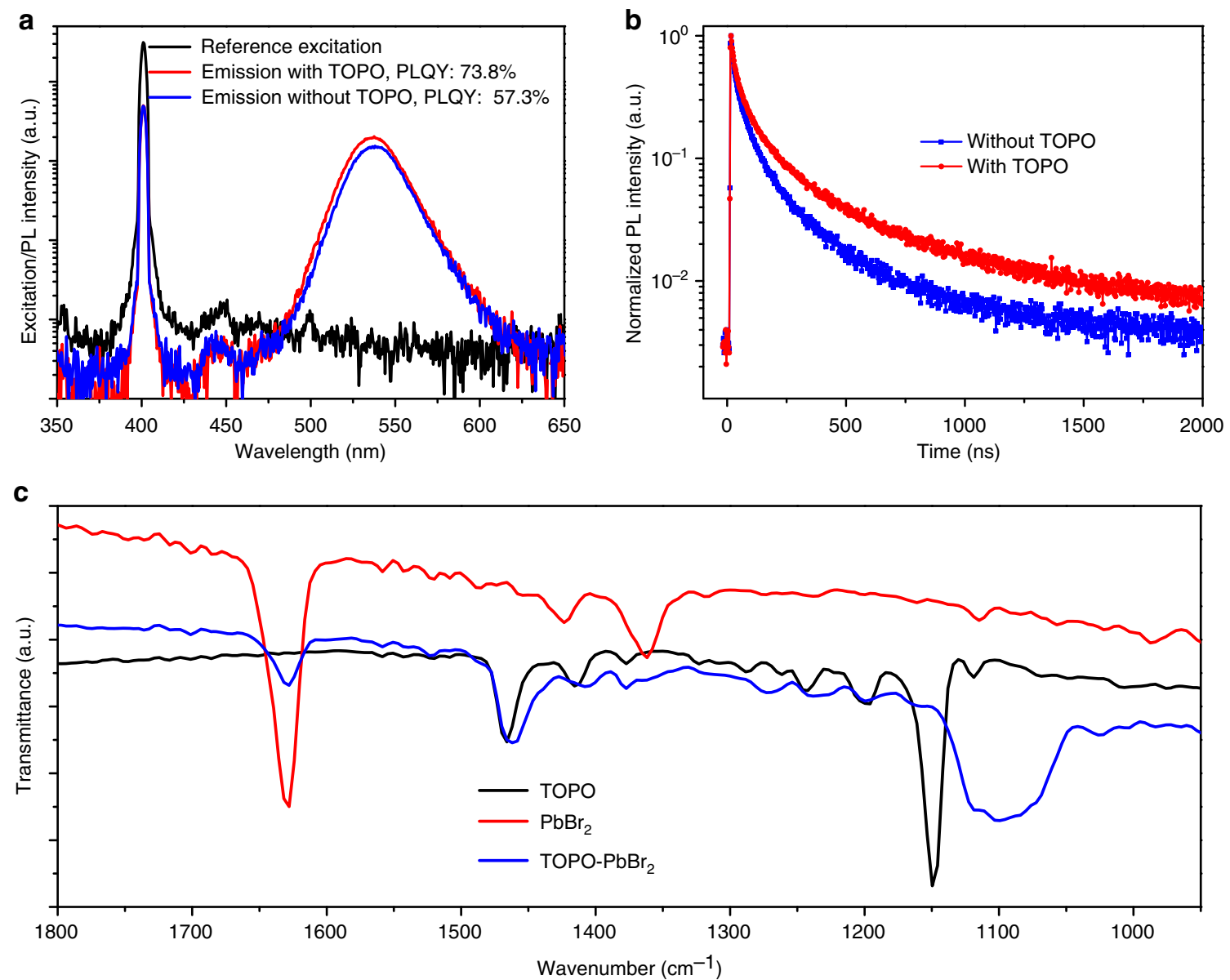

Fig. 2 Passivation effect of TOPO on the perovskite with $n=3$ composition. a Photoluminescence quantum yield $(\mathrm{PLQY})$ of the $P E A_{2}\left(F A P b B r_{3}\right)_{n-1} \mathrm{PbBr}_{4}(n$ $=3$ composition) perovskite films with and without TOPO passivation. $\mathbf{b}$ Time-resolved photoluminescence $(\mathrm{TRPL})$ of the $\mathrm{PEA} \mathrm{A}_{2}(\mathrm{FAPbBr})_{n-1} \mathrm{PbBr}_{4}(n=3$ composition) films with and without TOPO passivation layer. c Fourier transform infrared (FTIR) spectroscopy measurement for $\mathrm{TOPO} \mathrm{PbBr}_{2}$ and $\mathrm{TOPO}$ $\mathrm{PbBr}_{2}$ films prepared on silicon wafers

The passivation of halide perovskites is ascribed to the chemical interaction between molecular ligands and incomplete $\mathrm{PbI}_{6}$-octahedra in previous study ${ }^{39}$. To confirm this, Fourier transform infrared (FTIR) spectroscopy measurements were carried out, and the result is shown in Fig. 2c. An absorption peak located at $1150 \mathrm{~cm}^{-1}$ is observed for TOPO, which corresponds to $\mathrm{P}=\mathrm{O}$ bond stretching vibrations ${ }^{40}$. This $\mathrm{P}=\mathrm{O}$ bond absorption peak shifts to approximately $1100 \mathrm{~cm}^{-1}$ in the film comprised of TOPO and $\mathrm{PbBr}_{2}$, indicating that the bonding between perovskite and TOPO could be formed ${ }^{41,42}$.

Device structure and performance. We configure PeLEDs as the structure glass/ITO/PEDOT:PSS/PEA $2\left(\mathrm{FAPbBr}_{3}\right)_{n-1} \mathrm{PbBr}_{4} / \mathrm{TPBi} /$ LiF/Al. Modified PEDOT:PSS (m-PEDOT:PSS) is used as the hole injection layer (HIL), TPBi/LiF as the electron injection layer (EIL) and the quasi-2D perovskites as the emitting layer. The band alignment for all function layers is shown in Fig. 3a, and TOPO shown in the figure acts as a thin passivation layer on the perovskite film. Br-based perovskites usually exhibit a deeper valence band (approximately $-6.0 \mathrm{eV}$, Supplementary Fig.5) than that of PEDOT:PSS (approximately $-5.2 \mathrm{eV}$ ). We doped PSS-Na into PEDOT:PSS to increase the work function ${ }^{43}$, which can improve hole injection as well as device performance (Supplementary Fig.13). The cross-section scanning electron microscopy (SEM) image of the completed device is shown in Fig. 3b, and a clear sandwich device structure is observed. The thicknesses of $\mathrm{m}$ PEDOT:PSS (HIL), the perovskite layer and TPBi (EIL) are about
50, 110 and $30 \mathrm{~nm}$, respectively. The typical EL spectra of the $n=$ 3 composition-based PeLEDs under different voltage bias are shown in Fig. 3c. Although the perovskite films exhibit a mixture of many phases with different bandgaps, only single green EL is observed, even in logarithmic scale (Supplementary Fig. 8), which is different from the PL. It can be estimated that the charge density injected by EL $\left(10^{17} \mathrm{~s}^{-1} \mathrm{~cm}^{-2}\right)$ is two orders higher than that excited by PL $\left(10^{15} \mathrm{~s}^{-1} \mathrm{~cm}^{-2}\right)$ under injection/excitation of $5.1 \mathrm{~V}, 30 \mathrm{~mA} \mathrm{~cm}^{-2}$ and $1 \mathrm{~mW} \mathrm{~cm}^{-2}, 340 \mathrm{~nm}$ excitation, respectively, while the blue emission is still not observed in EL. This could be due to the different working mechanism between EL and PL. For the PL, the photo-excited charges are driven from a larger bandgap phase to a smaller bandgap phase by their conduction/ valence band energy difference. However, for EL, the driving force is not only the energy difference, but also the applied external electric field. As a result, most charges will be injected and recombined in the smallest band gap phases. This could be the reason that only green EL is observed even under high charge density injection. The EL peak is located at $532 \mathrm{~nm}$, and the full width at half maximum is about $23 \mathrm{~nm}$. The emission of our PeLEDs exhibited a good color purity $(95 \%)$ in the green region, with Commission Internationale de l'Eclairage (CIE) chromaticity coordinate at $(0.21,0.75)$ (Fig. 3d).

We characterize the PeLEDs with different compositions (Fig. 4a, b and Supplementary Fig. 9). It is found that the $n=2$ composition showed the lowest injection current (Supplementary Fig. 9), which could be due to the poor transport properties of 2D 
a

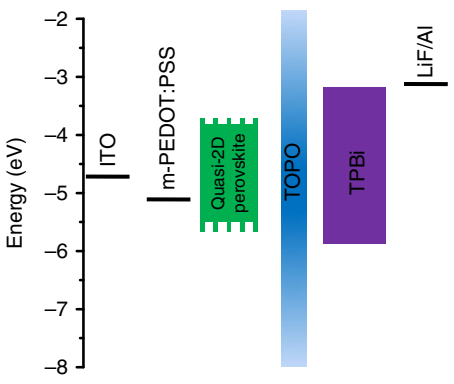

C

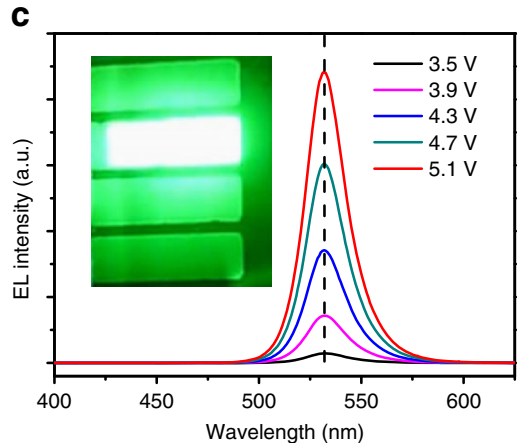

b

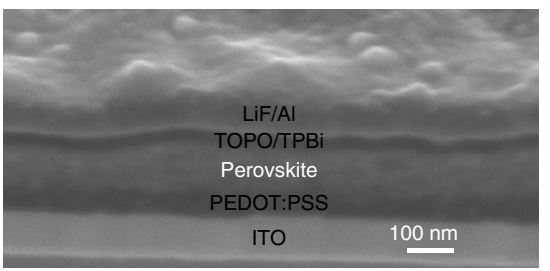

d

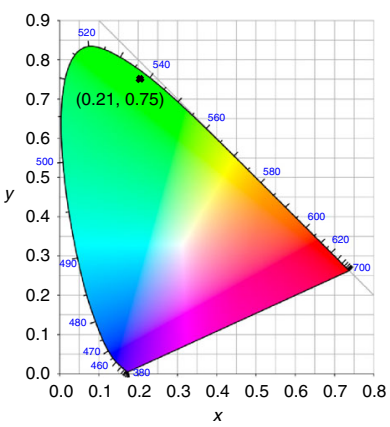

Fig. 3 Perovskite light-emitting diodes structure and electroluminescence. a Band alignment of each function layer in the devices. $\mathbf{b}$ Cross-section scanning electron microscopy (SEM) image of the device, the scale bar is $100 \mathrm{~nm}$. c Typical electroluminescence (EL) spectra of $\mathrm{PEA}_{2}\left(\mathrm{FAPbBr}_{3}\right)_{n-1} \mathrm{PbBr}_{4}(n=3$ composition) based PeLEDs under different voltage bias. Inset shows the electroluminescence image of PeLEDs. d The corresponding Commission Internationale de I'Eclairage (CIE) coordinate of typical PeLEDs based on $\mathrm{PEA}_{2}\left(\mathrm{FAPbBr}_{3}\right)_{n-1} \mathrm{PbBr}_{4}(n=3$ composition)

phase. Correspondingly, the brightness and $\mathrm{CE}$ of $n=2$ composition-based devices are both the lowest (Fig. 4a, b). The compositions of $n=3$ and $n=4$ based PeLEDs show the maximum brightness of about $7000 \mathrm{~cd} \mathrm{~m}^{-2}$ and $8700 \mathrm{~cd} \mathrm{~m}^{-2}$, respectively, while $n=3$ composition shows a higher CE $(40.2 \mathrm{~cd}$ $\mathrm{A}^{-1}$ ) than $n=4$ composition $\left(37.6 \mathrm{~cd} \mathrm{~A}^{-1}\right)$. For the $n=5$ and $n=6$ compositions, consistent with their PL efficiency (Fig.1c, e), their EL also could not compare with that of $n=3$ composition. The detailed device performances for various compositions are summarized in Table 1.

We next discuss the role of surface passivation in performance improvement for $n=3$ composition. It is found that the turn-on voltage increases and the injection current decreases after introducing a TOPO layer (Supplementary Fig.10). This is due to the insulating property of TOPO and indicates that the TOPO is successfully coated on the perovskite surface. Figure $4 \mathrm{c}$ shows the brightness curve of the PeLEDs with and without TOPO. The maximum brightnesses of the PeLEDs with and without passivation are at the same level, while the devices with passivation showed higher CE (Fig. 4d). The best performing device without TOPO passivation shows a $\mathrm{CE}$ of $52.5 \mathrm{~cd} \mathrm{~A}^{-1}$, while TOPO-treated devices show a $\mathrm{CE}$ as high as $62.4 \mathrm{~cd} \mathrm{~A}^{-1}$, and the power efficiency also increased from $49.4 \mathrm{~lm} \mathrm{~W}^{-1}$ to 53.3 $\mathrm{lm} \mathrm{W}^{-1}$ (Supplementary Fig.11). Accordingly, a high EQE of $14.36 \%$ is obtained (Fig. 4e). We also find that our devices show good reproducibility. The EQE histogram for 60 devices from five batches are presented, showing an average EQE of $13.1 \%$ with a low relative standard deviation of $4.4 \%$ (Fig. $4 \mathrm{f}$ ).

\section{Discussion}

Device stability is still a critical and common issue in PeLEDs. The stability of our PeLEDs with encapsulation was tested under a constant injection current in dry air (Supplementary Fig. 14). The luminance and CE increased at the beginning for several minutes, which could be due to trap filling ${ }^{15}$ that results in the improvement of electrical contacts. After that, the device performance gradually decreased, and finally the devices work for approximately $120 \mathrm{~min}$. We also noticed that larger injection current induces faster degradation, which could be due to heat accumulation or ion movements. Our device showed the almost same level stability of I-related perovskite with the stability in several $100 \mathrm{~min}^{15}$. Compared with the Br-related perovskite with several $10 \mathrm{~min}$ stability in previous reports ${ }^{15,44}$, our devices showed a little bit improvement.

Further improvement of PeLED stability is necessary, and we assume that there are several issues leading to PeLED instability. One major issue could be ion migration under voltage bias, and another issue could be the phase stability of perovskite materials under heating or moist conditions. Several strategies could be adopted to improve PeLED stability such as finding stable perovskite-emitting materials ${ }^{19,45}$, incorporating suitable groups to avoid or suppress the ion movements ${ }^{12,19}$, seeking effective ligands ${ }^{17,46}$ to bond with the grain surface and also the film surface to reduce Joule heating induced by non-radiative recombination and finding stable charge injection layers ${ }^{47}$.

In summary, by composition/phase engineering, we find that a quasi-2D perovskite of $\mathrm{PEA}_{2}\left(\mathrm{FAPbBr}_{3}\right)_{n-1} \mathrm{PbBr}_{4}$ with $n=3 \mathrm{com}$ position showed good PL. We also introduce surface passivation to reduce the non-radiative recombination on the perovskite surface or grain boundaries. Combining composition/phase engineering and surface passivation, high performance of quasi2D PeLEDs with a CE of $62.4 \mathrm{~cd} \mathrm{~A}^{-1}$ and an external quantum efficiency of $14.36 \%$ is achieved.

\section{Methods}

Materials. The modified PEDOT:PSS (m-PEDOT:PSS) solution is a mix of normal PEDOT:PSS (AI 4083) aqueous solution and $100 \mathrm{mg} \mathrm{ml}^{-1} \mathrm{PSS}-\mathrm{Na}$ (Sigma Aldrich) aqueous solution by a volume ratio of 6:5. TOPO, $\mathrm{PbBr}_{2}$, dimethyl sulfoxide (DMSO) and chlorobenzene (CB) were purchased from Sigma Aldrich. 2,2',2" 

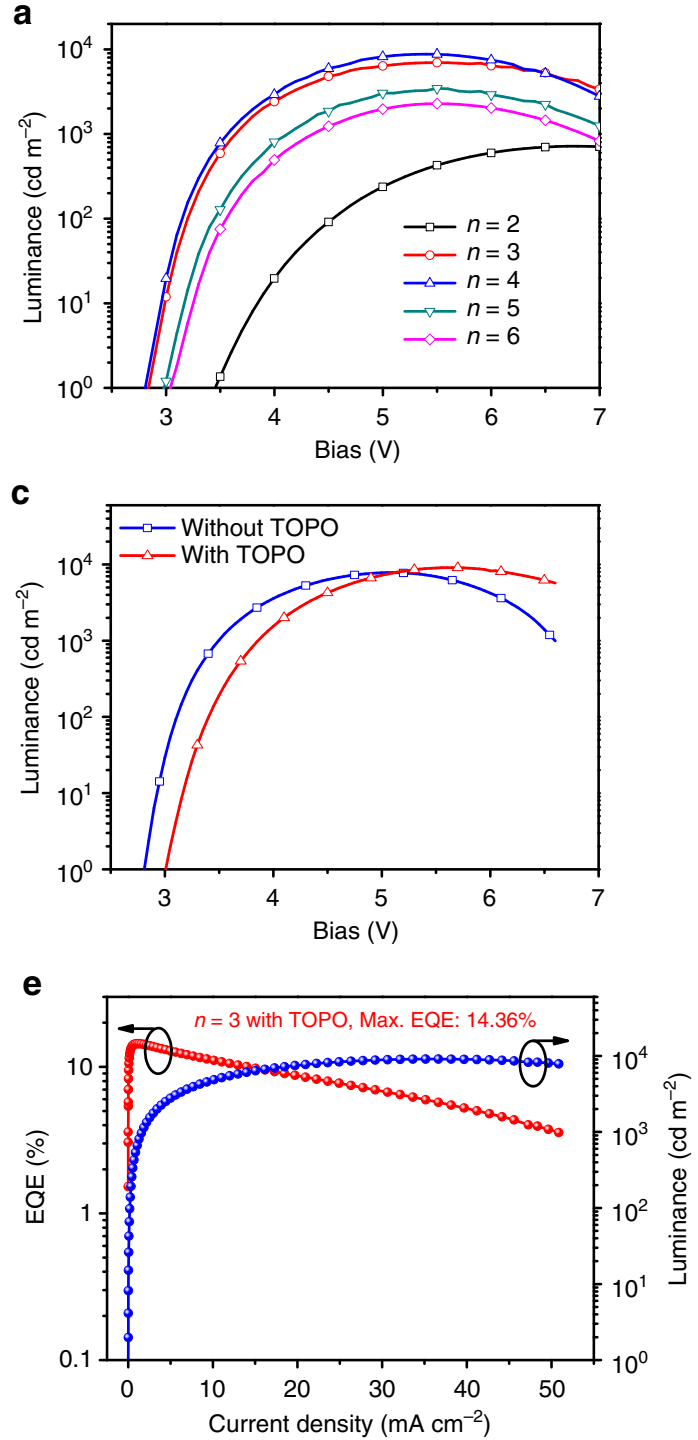
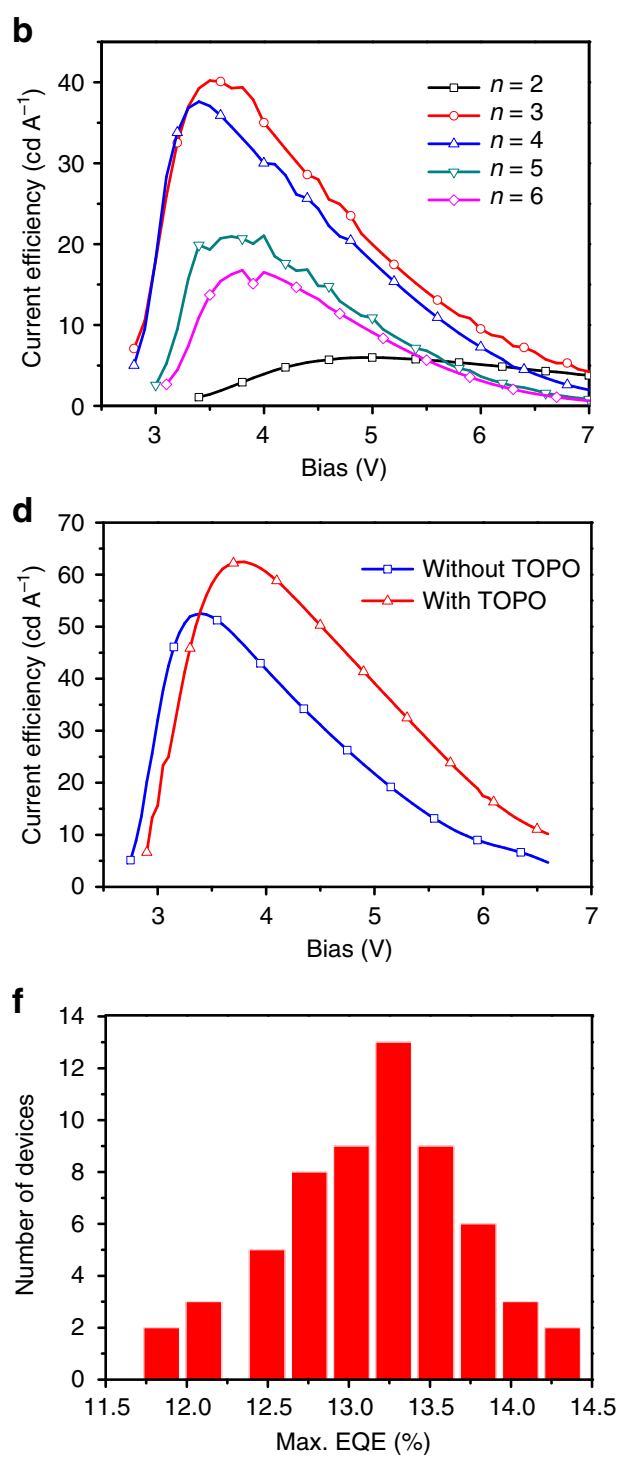

Fig. 4 Device performance of perovskite LEDs with different compositions and surface passivation. a Luminance-voltage (L-V) curves of $P E A_{2}\left(F A P b B r_{3}\right)_{n-}$ ${ }_{1} \mathrm{PbBr}_{4}$ with different compositions. b Current efficiency-voltage (CE-V) curves of $\mathrm{PEA}_{2}\left(\mathrm{FAPbBr}_{3}\right)_{n-1} \mathrm{PbBr}_{4}$ with different compositions. c Luminance-voltage $(\mathrm{L}-\mathrm{V})$ curves of $\mathrm{PEA}_{2}\left(\mathrm{FAPbBr}_{3}\right)_{n-1} \mathrm{PbBr}_{4}(n=3$ composition) devices with and without TOPO layer. $\mathbf{d}$ Current efficiency-voltage (CE-V) curves of $\mathrm{PEA}_{2}\left(\mathrm{FAPbBr}_{3}\right)_{n-1} \mathrm{PbBr}_{4}(n=3$ composition) devices with and without TOPO layer. e External quantum efficiency (EQE) of the champion device of $\mathrm{PEA}_{2}\left(\mathrm{FAPbBr}_{3}\right)_{n-1} \mathrm{PbBr}_{4}$ ( $n=3$ composition) with TOPO passivation layer. $\mathbf{f}$ Histogram of maximum EQEs measured from 60 devices

\begin{tabular}{|c|c|c|c|}
\hline Compositions & $\begin{array}{l}L_{\max }(c d \\
\left.\mathbf{m}^{-2}\right)\end{array}$ & $\begin{array}{l}\text { Current efficiency } \\
\left(\mathrm{cd} \mathrm{A}^{-1}\right)\end{array}$ & $\begin{array}{l}\text { Maximum EQE } \\
(\%)\end{array}$ \\
\hline$n=2$ & 717 & 5.98 & 1.54 \\
\hline$n=3$ & 6973 & 40.20 & 9.29 \\
\hline$n=3$ (best) & 7829 & 52.51 & 12.12 \\
\hline$n=4$ & 8779 & 37.61 & 8.45 \\
\hline$n=5$ & 3452 & 20.95 & 4.82 \\
\hline$n=6$ & 2281 & 16.77 & 4.00 \\
\hline$n=3$ and TOPO & 9120 & 62.43 & 14.36 \\
\hline
\end{tabular}

(1,3,5-Benzinetriyl)- tris(1-phenyl-1-H-benzimidazole) (TPBi) and formamidinium bromide (FABr) and methylammonium chloride (MACl) were purchased from Xi'an Polymer Light Technology Corp. Phenylethylammonium bromide (PEABr) was purchased from Dyesol (now Greatcell Solar).
Device fabrication. The indium tin oxide (ITO)-coated glass substrates were sequentially cleaned in detergent, distilled water, acetone and isopropanol by sonication and used as anode. The cleaned substrates were ultraviolet ozone treated for $15 \mathrm{~min}$ to make the surface hydrophilic, then m-PEDOT:PSS (or normal PEDOT:PSS) aqueous solution was spin-coated at $9000 \mathrm{rpm}$ (or $2500 \mathrm{rpm}$ ) for $40 \mathrm{~s}$ and baked at $160^{\circ} \mathrm{C}$ for $15 \mathrm{~min}$ in ambient air. Thereafter, the substrates were transferred into a nitrogen-filled glove box, and the $0.6 \mathrm{moll}^{-1}\left(\mathrm{~Pb}^{2+}\right.$ concentration) perovskite solution in DMSO was spin-coated onto the m-PEDOT:PSS (or normal PEDOT:PSS) films at $3000 \mathrm{rpm}$ for $2 \mathrm{~min}$, and after spin coating for $40 \mathrm{~s}, 100 \mu \mathrm{l}$ of $\mathrm{CB}$ was poured onto the film for pinning the perovskite crystallization, followed by annealing on a hot plate at $90^{\circ} \mathrm{C}$ for $1 \mathrm{~h}$. The perovskite precursor solution was prepared according to Supplementary Table 1 . For surface passivation, $2 \mathrm{mg} \mathrm{ml}^{-1}$ TOPO in CB was dripped onto the perovskite film for a $1 \mathrm{~min}$ to let TOPO passivate the film surface enough and then spin-coated at $7000 \mathrm{rpm}$ for $1 \mathrm{~min}$. Finally, the fabrication of PeLEDs was completed by depositing TPBi, LiF (less than $1 \mathrm{~nm}$ ) and $\mathrm{Al}$ electrode layer by layer through a shadow mask in a high vacuum thermal evaporator. The device area was $0.108 \mathrm{~cm}^{2}$ as defined by the overlapping area of the ITO and Al electrode. The thickness of ITO and $\mathrm{Al}$ are $130 \mathrm{~nm}$ and 60 $\mathrm{nm}$, respectively.

Material and device characterizations. XRD measurements were performed with a Rigaku D/max $2500 \mathrm{H}$ equipment with a conventional $\mathrm{Cu}$ target X-ray tube $(\mathrm{Cu}$ 
K-alpha, $\lambda=1.5418 \AA$ ) set to $40 \mathrm{kV}$ and $200 \mathrm{~mA}$. Steady-state PL spectra and timeresolved fluorescence spectra (TRPL) of the perovskite films were measured at room temperature in the ambient air using a fluorescent spectrophotometer (HORIBA JY Nanolog-TCSPC). The excitation wavelength for PL and TRPL are both $340 \mathrm{~nm}$, which were provided by $450 \mathrm{~W}$ xenon lamp and a pulsed diode laser (NanoLed-340), respectively. The PLQY of the perovskite films was measured using an Edinburgh FLS920 equipment with a $450 \mathrm{~W}$ xenon lamp as excitation source. The following settings were applied for PLQY measurements: excitation wavelength of $400 \mathrm{~nm}$; bandpass values of 3.00 and $0.35 \mathrm{~nm}$ for the excitation and emission slits, respectively; step increments of $0.5 \mathrm{~nm}$ and integration time of $0.3 \mathrm{~s}$ per data point. We measured the light intensity with a laser power meter and the estimated light power density incident on the samples was about $3.5 \mathrm{~mW} \mathrm{~cm}^{-2}$. The ultraviolet-visible absorbance spectra were recorded on an ultraviolet-visible spectrophotometer (Cary 5000, Varian). The FTIR spectra were recorded on an infrared spectrophotometer (Excalibur 3100, Varian) in a general mode that did not use attenuated total reflection component. AFM images of the perovskite films were collected in noncontact mode (Bruker FASTSCANBIO). The cross-sectional image of PeLEDs was made on a scanning electron/focused ion beam double-beam equipment (FEI Nova200 NanoLab) operated at $2 \mathrm{kV}$. Ultraviolet photoelectron spectroscopy (UPS) spectra were collected on a Thermo Scientific ESCALab250Xi equipment with an applied bias of $-10 \mathrm{~V}$. He I ultraviolet radiation source $(21.22 \mathrm{eV})$ was used. The helium pressure in the analysis chamber during measurement was about $2 \mathrm{E}-8$ mbar. The film samples over the ITO layer had a conductive connection with an Au sample, so the Fermi level value of the film samples is equal to that of Au sample. The work function $\phi$ (that is the Fermi level absolute value of the free film) of the test films can be calculated from following equation: $h \nu-\phi=E_{\mathrm{Fermi}}-E_{\text {cutoff, }}$ where $E_{\mathrm{Fermi}}$ and $E_{\text {cutoff }}$ are respectively the value of Fermi level position and the steep edge position in the UPS spectrum of the test film, $h \nu=21.22 \mathrm{eV}, E_{\text {Fermi }}$ is $21.02 \mathrm{eV}$ for Au. Two Keithley 2400 source meter units linked to a calibrated silicon photodiode were used to measure the current-voltage-brightness characteristics. The measurement system was carefully calibrated by a Hamamatsu C9920-02G equipment and a $100 \mathrm{~mm}$ integrating sphere of Enli Technology using our perovskite LEDs. Lambertian profile was used in the calculation of EQE. All the device characterization tests of perovskite LEDs were carried out at room temperature in the ambient air for the un-encapsulated devices, except that operational stability test was carried in dry air for the encapsulated devices.

Data availability. The data that support the findings of this study are available from the corresponding author upon reasonable request.

Received: 13 September 2017 Accepted: 11 January 2018

Published online: 08 February 2018

\section{References}

1. Deschler, F. et al. High photoluminescence efficiency and optically pumped lasing in solution-processed mixed halide perovskite semiconductors. J. Phys. Chem. Lett. 5, 1421-1426 (2014).

2. Xing, G. et al. Low-temperature solution-processed wavelength-tunable perovskites for lasing. Nat. Mater. 13, 476-480 (2014).

3. Protesescu, L. et al. Nanocrystals of cesium lead halide perovskites $\left(\mathrm{CsPbX}_{3}\right.$, $\mathrm{X}=\mathrm{Cl}, \mathrm{Br}$, and I): novel optoelectronic materials showing bright emission with wide color gamut. Nano Lett. 15, 3692-3696 (2015).

4. Zhang, F. et al. Brightly luminescent and color-tunable colloidal $\mathrm{CH}_{3} \mathrm{NH}_{3} \mathrm{PbX}_{3}$ $(\mathrm{X}=\mathrm{Br}, \mathrm{I}, \mathrm{Cl})$ quantum dots: potential alternatives for display technology. ACS Nano 9, 4533-4542 (2015).

5. Song, J. et al. Quantum dot light-emitting diodes based on inorganic perovskite cesium lead halides $\left(\mathrm{CsPbX}_{3}\right)$. Adv. Mater. 27, 7162-7167 (2015).

6. Protesescu, L. et al. Monodisperse formamidinium lead bromide nanocrystals with bright and stable green photoluminescence. J. Am. Chem. Soc. 138, 14202-14205 (2016).

7. Levchuk, I. et al. Brightly luminescent and color-tunable formamidinium lead halide perovskite $\mathrm{FAPbX}_{3}(\mathrm{X}=\mathrm{Cl}, \mathrm{Br}, \mathrm{I})$ colloidal nanocrystals. Nano Lett. 17, 2765-2770 (2017).

8. Kovalenko, M. V. et al. Properties and potential optoelectronic applications of lead halide perovskite nanocrystals. Science 358, 745-750 (2017).

9. Tan, Z. K. et al. Bright light-emitting diodes based on organometal halide perovskite. Nat. Nanotech. 9, 687-692 (2014).

10. Wang, J. et al. Interfacial control toward efficient and low-voltage perovskite light-emitting diodes. Adv. Mater. 27, 2311-2316 (2015).

11. Cho, H. et al. Overcoming the electroluminescence efficiency limitations of perovskite light-emitting diodes. Science 350, 1222-1225 (2015).

12. Zhang, L. et al. Ultra-bright and highly efficient inorganic based perovskite light-emitting diodes. Nat. Commun. 8, 15640 (2017).
13. Yuan, M. J. et al. Perovskite energy funnels for efficient light-emitting diodes. Nat. Nanotech. 11, 872-877 (2016)

14. Wang, N. et al. Perovskite light-emitting diodes based on solution-processed self-organized multiple quantum wells. Nat. Photon. 10, 699-704 (2016).

15. Xiao, Z. G. et al. Efficient perovskite light-emitting diodes featuring nanometre-sized crystallites. Nat. Photon. 11, 108-115 (2017).

16. Sutherland, B. R. \& Sargent, E. H. Perovskite photonic sources. Nat. Photon 10, 295-302 (2016).

17. Veldhuis, S. A. et al. Perovskite materials for light-emitting diodes and lasers. Adv. Mater. 28, 6804-6834 (2016)

18. Kim, Y. H., Cho, H. \& Lee, T. W. Metal halide perovskite light emitters. Proc Natl. Acad. Sci. USA 113, 11694-11702 (2016).

19. Ling, Y. et al. Enhanced optical and electrical properties of polymer-assisted all-inorganic perovskites for light-emitting diodes. Adv. Mater. 28, 8983-8989 (2016).

20. Era, M. et al. Electroluminescent device using two dimensional semiconductor $\left(\mathrm{C}_{6} \mathrm{H}_{5} \mathrm{C}_{2} \mathrm{H}_{4} \mathrm{NH}_{3}\right)_{2} \mathrm{PbI}_{4}$ as an emitter. Synth. Met. 71, 2013-2014 (1995).

21. Dou, L. et al. Atomically thin two-dimensional organic-inorganic hybrid perovskites. Science 349, 1518-1521 (2015).

22. Smith, I. C. et al. A layered hybrid perovskite solar-cell absorber with enhance moisture stability. Angew. Chem. Int. Ed. 53, 11232-11235 (2014).

23. Tsai, H. et al. High-efficiency two-dimensional Ruddlesden-Popper perovskite solar cells. Nature 536, 312-316 (2016).

24. Quan, L. N. et al. Ligand-stabilized reduced-dimensionality perovskites. J. Am. Chem. Soc. 138, 2649-2655 (2016).

25. Byun, J. et al. Efficient visible quasi-2D perovskite light-emitting diodes. $A d v$ Mater. 28, 7515-7520 (2016).

26. Reineke, S. et al. White organic light-emitting diodes with fluorescent tube efficiency. Nature 459, 234-238 (2009).

27. Pimputkar, S. et al. Prospects for LED lighting. Nat. Photon. 3, 180-182 (2009).

28. Noel, N. K. et al. Enhanced photoluminescence and solar cell performance via Lewis base passivation of organic-inorganic lead halide perovskites. ACS Nano 8, 9815-9821 (2014).

29. Wang, F. et al. Phenylalkylamine passivation of organolead halide perovskites enabling high-efficiency and air-stable photovoltaic cells. Adv. Mater. 28, 9986-9992 (2016).

30. de Quilettes, D. W. et al. Impact of microstructure on local carrier lifetime in perovskite solar cells. Science 348, 683-686 (2015).

31. de Quilettes, D. W. et al. Photoluminescence lifetimes exceeding $8 \mu$ s and quantum yields exceeding $30 \%$ in hybrid perovskite thin films by ligand passivation. ACS Energy Lett. 1, 438-444 (2016).

32. Jeon, N. J. et al. Solvent engineering for high-performance inorganic-organic hybrid perovskite solar cells. Nat. Mater. 13, 897-903 (2014).

33. You, J. et al. Low-temperature solution-processed perovskite solar cells with high efficiency and flexibility. ACS Nano 8, 1674-1680 (2014).

34. Stranks, S. D. et al. Electron-hole diffusion lengths exceeding 1 micrometer in an organometal trihalide perovskite absorber. Science 342, 341-344 (2013)

35. Meng, L. et al. Pure formamidinium-based perovskite light-emitting diodes with high efficiency and low driving voltage. Adv. Mater. 29, 1603826 (2017).

36. Quan, L. N. et al. Tailoring the energy landscape in quasi-2D halide perovskites enables efficient green-light emission. Nano. Lett. 17, 3701-3709 (2017).

37. Liang, D. et al. Color-pure violet-light-emitting diodes based on layered lead halide perovskite nanoplates. ACS Nano 10, 6897-6904 (2016).

38. Arora, N. et al. Photovoltaic and amplified spontaneous emission studies of high-quality formamidinium lead bromide perovskite films. Adv. Funct. Mater. 26, 2846-2854 (2016).

39. Bi, D. Q. et al. Polymer-templated nucleation and crystal growth of perovskite films for solar cells with efficiency greater than $21 \%$. Nat. Energy 1, 16142 (2016).

40. Bayou, N. et al. Elaboration and characterisation of a plasticized cellulose triacetate membrane containing trioctylphosphine oxyde (TOPO): application to the transport of uranium and molybdenum ions. C. R. Chim. 13, 1370-1376 (2010).

41. Söderlind, F. et al. Synthesis and characterisation of $\mathrm{Gd}_{2} \mathrm{O}_{3}$ nanocrystals functionalised by organic acids. J. Colloid Interface Sci. 288, 140-148 (2005).

42. Chang, S. M., Lo, P. H. \& Chang, C. T. Photocatalytic behavior of TOPOcapped $\mathrm{TiO}_{2}$ nanocrystals for degradation of endocrine disrupting chemicals. Appl. Catal. B Environ. 91, 619-627 (2009).

43. Zuo, C. T. \& Ding, L. M. Modified PEDOT layer makes a $1.52 \mathrm{~V} \mathrm{~V}_{\mathrm{OC}}$ for perovskite/PCBM solar cells. Adv. Energy Mater. 7, 1601193 (2016).

44. Zhang, X. et al. Enhancing the brightness of cesium lead halide perovskite nanocrystal based green light-emitting devices through the interface engineering with perfluorinated ionomers. Nano. Lett. 16, 1415-1420 (2016)

45. Kulbak, M., Cahen, D. \& Hodes, G. How important is the organic part of lead halide perovskite photovoltaic cells? Efficient $\mathrm{CsPbBr} 3$ cells. J. Phys. Chem. Lett. 6, 2452-2456 (2015). 
46. Li, J. et al. 50-Fold EQE improvement up to $6.27 \%$ of solution-processed allinorganic perovskite $\mathrm{Cs} \mathrm{PbBr}_{3}$ QLEDs via surface ligand density control. $A d v$. Mater. 29, 1603885 (2017).

47. You, J. et al. Improved air stability of perovskite solar cells via solutionprocessed metal oxide transport layers. Nat. Nanotech. 11, 75-81 (2016).

\section{Acknowledgements}

This work is supported by National Natural Science Foundation of China (Grant Numbers: 61574133, 61634001), National Key Research and Development Program of China (Grant No. 2016YFB0700700), National 1000 Young Talents awards and Young top-notch talent project of Beijing.

\section{Author contributions}

J.Y. conceived the idea, designed the experiment and analyzed the data. X.Y. fabricated devices and collected all data. X.Z., J.D., Z.C., Q.J., J.M., P.W., L.Z. and Z.Y. were involved in data analysis. J.Y. and X.Y. co-wrote the manuscript. J.Y. directed and supervised the project. All authors contributed to discussions and finalization of the manuscript.

\section{Additional information}

Supplementary Information accompanies this paper at https://doi.org/10.1038/s41467018-02978-7.
Competing interests: The authors declare no competing financial interests.

Reprints and permission information is available online at http://npg.nature.com/ reprintsandpermissions/

Publisher's note: Springer Nature remains neutral with regard to jurisdictional claims in published maps and institutional affiliations.

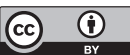

Open Access This article is licensed under a Creative Commons Attribution 4.0 International License, which permits use, sharing, adaptation, distribution and reproduction in any medium or format, as long as you give appropriate credit to the original author(s) and the source, provide a link to the Creative Commons license, and indicate if changes were made. The images or other third party material in this article are included in the article's Creative Commons license, unless indicated otherwise in a credit line to the material. If material is not included in the article's Creative Commons license and your intended use is not permitted by statutory regulation or exceeds the permitted use, you will need to obtain permission directly from the copyright holder. To view a copy of this license, visit http://creativecommons.org/ licenses/by/4.0/.

(C) The Author(s) 2018 